05

\title{
Температурное поведение антиферромагнитной восприимчивости нано-ферригидрита из измерений кривых намагничивания в полях до $250 \mathrm{kOe}$
}

\author{
() Д.А. Балаев ${ }^{1,2}$, С.И. Попков ${ }^{1,2}$, А.А. Красиков ${ }^{1,2}$, А.Д. Балаев ${ }^{1}$, А.А. Дубровский ${ }^{1}$, \\ С.В. Столяр ${ }^{1,2}$, Р.Н. Ярославцев ${ }^{1,2}$, В.П. Ладыгина ${ }^{3}$, Р.С. Исхаков ${ }^{1}$ \\ ${ }^{1}$ Институт фризики им. Л.В. Киренского СО РАН ФИЦ КНЦ СО РАН, \\ Красноярск, Россия \\ ${ }^{2}$ Сибирский Федеральный университет, \\ Красноярск, Россия \\ ${ }_{3}^{3}$ Президиум Красноярского научного центра ФИЦ КНЦ СО РАН, \\ Красноярск, Россия \\ E-mail: dabalaev@iph.krasn.ru
}

(Поступила в Редакцию 4 апреля 2017 г.)

Рассмотрена проблема кроссовинга температурной зависимости антиферромагнитной восприимчивости наночастиц ферригидрита. Атомы железа $\mathrm{Fe}^{3+}$ в ферригидрите упорядочены антиферромагнитно, однако наличие дефектов на поверхности и в объеме наночастиц индуцирует нескомпенсированный магнитный момент, что приводит к типичному суперпарамагнитному поведению ансамбля наночастиц с характерной температурой блокировки. В разблокированном состоянии кривые намагничивания таких объектов описываются как суперпозиция функции Ланжевена и линейного по полю вклада от антиферромагнитной „сердцевины“ частиц. По результатам большого количества исследований кривых намагничивания, проводимых на наночастицах ферригидрита (и родственного ему ферритина) в диапазоне полей до $60 \mathrm{kOe}$, зависимость $\chi_{\mathrm{AF}}(T)$ убывает с ростом температуры, что связывалось ранее с эффектом суперантиферромагнетизма. При увеличении диапазона магнитных полей до $250 \mathrm{kOe}$ значения $\chi_{\mathrm{AF}}$, получаемые из анализа кривых намагничивания, становятся меньшими по величине, однако изменяется характер температурной эволюции $\chi_{\mathrm{AF}}$ : теперь зависимость $\chi_{\mathrm{AF}}(T)$ - возрастающая функция. Последнее типично для системы AF частиц со случайной ориентацией кристаллографических осей. Для корректного определения антиферромагнитной восприимчивости AF наночастиц (по крайней мере, ферригидрита) и поиска эффектов, связанных с эффектом суперантиферромагнетизма, необходимо в эксперименте использовать диапазон полей, значительно превышающий используемый в большинстве работ стандартное значение $60 \mathrm{kOe}$. Анализ температурной эволюции кривых намагничивания показал, что обнаруженный кроссовер обусловлен наличием малых магнитных моментов в образцах.

Исследование выполнено при финансовой поддержке Российского фонда фундаментальных исследований, Правительства Красноярского края, Красноярского краевого фонда поддержки научной и научно-технической деятельности в рамках научных проектов № 17-42-240138, 17-43-240527.

DOI: 10.21883/FTT.2017.10.44959.115

\section{1. Введение}

Несмотря на большое количество работ по исследованию магнитных наночастиц, ввиду постоянно расширяющихся областей их практического применения, многие наблюдаемые свойства таких объектов остаются необъясненными к настоящему времени. К ним относится проблема описания кривой намагничивания наноразмерных частиц материалов, которые в объемном виде обладают антиферромагнитным (AF) порядком [1-6]. В таких наночастицах, как правило, сохраняется AF порядок в „сердцевине“(,ядре“ фекты и дефекты приводят к появлению нескомпенсированного магнитного момента частиц $\mu_{P}[6-11]$, а наличие оборванных химических связей в присутствии $\mathrm{AF}$ взаимодействий может являться причиной спинстекольного поведения поверхностных атомов [10-17].
В области температур выше температуры суперпарамагнитной (SP) блокировки $T_{B}$ кривая намагничивания $\mathrm{M}(\mathrm{H}) \mathrm{AF}$ частиц описывается выражением

$$
\mathrm{M}(\mathrm{H})=M_{0} L\left(\mu_{P}, H\right)+\chi_{\mathrm{AF}} H .
$$

Первое слагаемое выражения (1) соответствует процессам выстраивания нескомпенсированных магнитных моментов по направлению внешнего поля, которые описываются классической функцией Ланжевена $L\left(\mu_{P} H\right)=\operatorname{coth}\left(\mu_{P}, H / k T\right)-1 /\left(\mu_{P}, H / k T\right)\left(M_{0}-\right.$ намагниченность насыщения). Второе слагаемое выражения (1) описывает скос подрешеток $\mathrm{AF}$ „ядра“ частиц во внешнем поле; $\chi_{\mathrm{AF}}-\mathrm{AF}$ восприимчивость.

Во многих случаях корректное описание экспериментальных зависимостей $\mathrm{M}(\mathrm{H})$ AF частиц требует учета функции распределения по магнитным моментам частиц [8,15-19]. В этом случае выражение (1) можно 
переписать в виде

$$
\mathrm{M}(\mathrm{H})=N_{P} \int_{\mu_{\min }}^{\mu_{\max }} L\left(\mu_{P}, H\right) f\left(\mu_{P}\right) \mu_{p} d \mu_{P}+\chi_{\mathrm{AF}} H .
$$

Здесь $N_{P}$ - количество частиц в единице массы образца, $f\left(\mu_{P}\right)$ - функция распределения по магнитным моментам частиц. При описании экспериментальных данных выражениями (1), (2) можно получить величины среднего магнитного момента частиц $\left\langle\mu_{P}\right\rangle$, значения $\chi_{\mathrm{AF}}$, а также их температурную эволюцию. В большинстве случаев для антиферромагнитно упорядоченного ферригидрита (а также ферритина), о котором и пойдет речь в данной работе, оказывалось, что температурная зависимость $\chi_{\mathrm{AF}}(T)$ - убывающая с ростом температуры функция [8,15,18-27], что в корне противоречит стандартному поведению антиферромагнитной восприимчивости объемного антиферромагнетика со случайной ориентацией кристаллографических осей в области температур, меньших температуры Нееля (как в случае поликристалла, где величина $\chi_{\mathrm{AF}}$ растет с увеличением температуры). Наблюдаемое „аномальное“ поведение зависимости $\chi_{\mathrm{AF}}(T)$ в наночастицах зачастую „списывалось“ на влияние эффекта суперантиферромагнетизма.

Этот эффект был предсказан Неелем [28,29] и заключается в том, что в AF частицах с четным числом ферромагнитных плоскостей поверхностные спины под действием приложенного поля (Н перпендикулярно оси легкого намагничивания) подворачиваются в большей степени, чем спины „внутренних плоскостей“. Заметное увеличение AF восприимчивости может наблюдаться для частиц, имеющих в диаметре не более нескольких десятков ферромагнитно упорядоченных плоскостей, что и имеет место для частиц, исследовавшихся в работах $[8,15,18-27]$.

Однако в подавляющем большинстве работ по наночастицам ферригидрита и ферритину используемый диапазон внешних магнитных полей обычно ограничивается значением $\sim 60 \mathrm{kOe}$. И в этом диапазоне полей при температурах выше $\sim 100 \mathrm{~K}$ зависимость $\mathrm{M}(\mathrm{H})$, предсказываемая функцией Ланжевена для магнитных моментов порядка $10-30 \mu_{B}\left(\mu_{\mathrm{B}}-\right.$ магнетон Бора), близка к линейной зависимости. Это обстоятельство вносит неоднозначность в процедуру обработки экспериментальных кривых намагничивания $\mathrm{M}(\mathrm{H})$ выражениями (1) и (2) ввиду одинаковой функциональной зависимости для малых частиц $\left(\mu_{P} \sim 10-30 \mu_{B}\right)$ и „отклику“ АF-упорядоченного ,ядра““частиц [второй член выражений (1), (2)]. Следовательно, для более корректного определения значений $\chi_{\mathrm{AF}}$ по выражениям (1), (2) целесообразно расширить диапазон внешних магнитных полей при экспериментальном исследовании намагничивания AF наночастиц.

В работе [4] было показано, что „аномальное“ уменьшение $\chi_{\mathrm{AF}}$ с ростом $T$, наблюдавшееся для AF-упорядоченного ферритина и получаемое из обработки зависимостей $\mathrm{M}(\mathrm{H})$ в диапазоне до $60 \mathrm{kOe}$, может быть связано не с эффектом суперантиферромагнетизма, а являться следствием влияния малых магнитных моментов частиц, либо магнитной анизотропии.

В работе [30] показано, что результаты анализа зависимости $\mathrm{M}(\mathrm{H})$ синтетического ферригидрита (размер $\sim 5.5 \mathrm{~nm})$ по выражениям типа (1) и (2) зависят от диапазона магнитного поля. При обработке экспериментальных данных в полях до $120 \mathrm{kOe}$ величины $\chi_{\mathrm{AF}}$ оказываются несколько меньше, чем для диапазона до 40-60 kOе (при $T=100 \mathrm{~K})$.

В настоящей работе для исследования процессов высокополевой намагниченности образцов ферригидрита была использована методика импульсных магнитных полей напряженностью до $250 \mathrm{kOe}$. Используемый ферригидрит (номинальная формула $5 \mathrm{Fe}_{2} \mathrm{O}_{3} \cdot 9 \mathrm{H}_{2} \mathrm{O}$ ) продукт жизнедеятельности бактерий, так называемый бактериальный ферригидрит [31]. Ранее было показано, что низкотемпературный отжиг исходного образца приводит к укрупнению частиц и, как следствие, к увеличению их суперпарамагнитной температуры блокировки $T_{B}$ [32] и нескомпенсированного магнитного момента $\mu_{P}$ [33], что дает возможность исследования свойств наночастиц ферригидрита, различающихся средним размером [34,35]. Анализ кривых намагничивания по выражению (2) в температурном интервале $T>T_{B}$ в диапазоне полей до $60 \mathrm{kOe}$ показал, что и для бактериального ферригидрита зависимость $\chi_{\mathrm{AF}}(T)$ - убывающая с ростом температуры функция. С одной стороны, это согласуется с результатами других исследований ферригидрита $[19,21,22,24-27]$ и ферритина $[15,18,20,23]$ (в ферритине частицы ферригидрита заключены в белковую оболочку). С другой стороны, для более корректного выяснения поведения $\chi_{\mathrm{AF}}(T)$ необходимо исследовать кривые намагничивания в более широком диапазоне полей [14]. Измерения в диапазоне полей до $250 \mathrm{kOe}$ мы провели на двух образцах бактериального ферригидрита из серии, исследованной в работе [33].

\section{2. Эксперимент}

2.1. Получение и характеризация образцов. Получение нано-ферригидрита, образующегося в результате жизнедеятельности бактерий Klebsiella oxytoca, описано в работах [6,31]. Исследованные в настоящей работе образцы были подвергнуты дополнительному низкотемпературному отжигу в воздушной атмосфере при $140^{\circ} \mathrm{C}$ в течение 3 и 240 часов и далее обозначены как $3 \mathrm{~h}$ и 240h. По совокупности данных просвечивающей электронной микроскопии и величины среднего магнитного момента $\left\langle\mu_{P}\right\rangle$, полученного из анализа кривых намагничивания в диапазоне до $60 \mathrm{kOe}$, средний размер частиц в этих образцах составляет 3.8 и $4.6 \mathrm{~nm}$ для образцов $3 \mathrm{~h}$ и 240h соответственно [33]. Для характеризации образцов была также использована и мёссбауэровская методика. Результаты обработки мёссбауэровских спектров показали хорошее согласие с параметрами модельных спектров, полученных ранее на ферригидрите [32,33], и показали, что при отжиге 
Параметры, использованные при подгонке экспериментальных данных (рис. 2,3) по выражениям (2) („большие“ частицы) и (4) („малые частицы“), а также размеры частиц $\langle d\rangle$ и $d_{\mathrm{SM}}$, оцененные по выражениям (5) и (6) соответственно

\begin{tabular}{c|c|c|c|c|c|c|c|c}
\hline \multirow{2}{*}{ Образец } & \multicolumn{3}{|c|}{ „Большие“ частицы, $f\left(\mu_{P}\right)$} & \multicolumn{3}{|c}{ „Малые“ частицы } \\
\cline { 2 - 9 } & $\left\langle\mu_{P}\right\rangle(T=0)$ & $\begin{array}{c}M_{S}(T=0) \\
\mathrm{emu} / \mathrm{g}\end{array}$ & $N_{P}$ & $\begin{array}{c}\langle d\rangle, \\
\mathrm{nm}\end{array}$ & $\mu_{\mathrm{SM}}$ & $\begin{array}{c}M_{\mathrm{SM}}, \\
\mathrm{emu} / \mathrm{g}\end{array}$ & $\begin{array}{c}N_{\mathrm{SM}} \\
\mathrm{nm}\end{array}$ \\
\hline $3 \mathrm{~h}$ & $230 \mu_{B}$ & 3.45 & $1.61 \cdot 10^{18}$ & 3.8 & $18 \mu_{B}$ & 5.8 & $3.5 \cdot 10^{19}$ & $<1$ \\
$240 \mathrm{~h}$ & $315 \mu_{B}$ & 4.65 & $1.59 \cdot 10^{18}$ & 4.6 & $40 \mu_{B}$ & 5.1 & $1.4 \cdot 10^{19}$ & $\sim 1$
\end{tabular}

не наблюдается особенностей, свидетельствующих о появлении новых фаз гидроксида или оксида железа.

2.2. Измерения магнитных свойств. Квазистатические магнитные измерения проводили на вибрационном магнетометре (VSM). Исследуемый порошок фиксировали в измерительной капсуле в парафине. Для измерений температурных зависимостей магнитного момента $\mathrm{M}(\mathrm{T})$ использовали режимы охлаждения без поля (ZFC — zero field cooling) и во внешнем поле (FC field cooling).

Измерения кривых намагничивания $\mathrm{M}(\mathrm{H})$ в импульсных магнитных полях проводили на установке, работающей в Институте физики им. Киренского СО РАН (Красноярск). Исследуемый образец (в виде порошка) надежно фиксировали в индукционном датчике импульсного магнитометра (далее PFM - Pulse Field Magnetometer). Длительность импульса (при изменении поля от $H=0 \rightarrow H_{\max } \rightarrow H=0$ ) составляла $16 \mathrm{~ms}$. Изотермы намагничивания измеряли при различных амплитудах импульса магнитного поля вплоть до $250 \mathrm{kOe}$. Данные, полученные с помощью методики PFM, в области полей до $60 \mathrm{kOe}$ хорошо совпадали с результатами измерений VSM.

\section{3. Результаты и обсуждение}

На рис. 1 приведены зависимости магнитного момента $M(T)$ от температуры исследованных образцов в

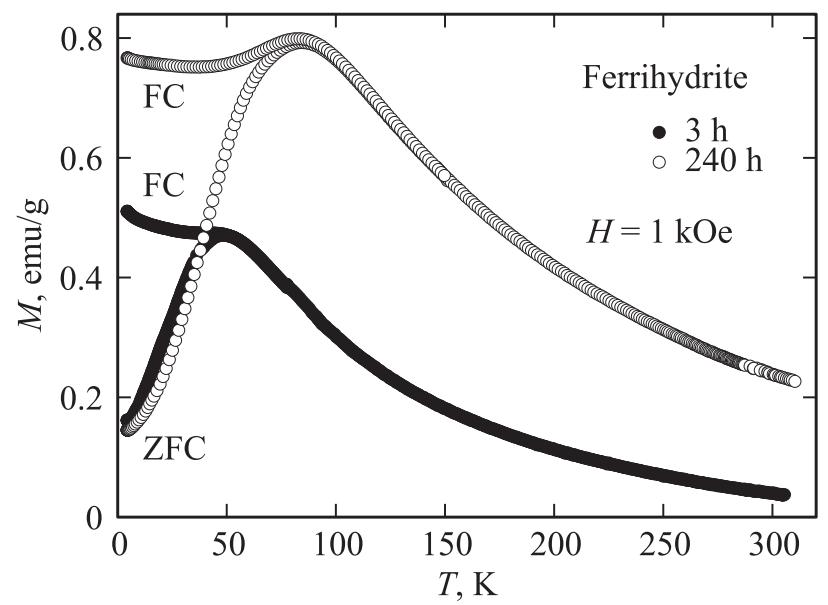

Рис. 1. Температурные зависимости магнитного момента $M(T)$ исследованных образцов ферригидрита в режимах ZFC и FC. режимах ZFC и FC. Видно, что зависимости $M(T)_{\mathrm{ZFC}}$ демонстрируют максимумы, соответствующие SP блокировке. Возрастание температуры блокировки с увеличением времени отжига коррелирует с ростом размеров и соответственно среднего магнитного момента частиц $\left\langle\mu_{P}\right\rangle[32,33,36]$.

На рис. 2, 3 представлены экспериментальные кривые намагничивания исследованных образцов при различных температурах, превышающих температуру блокировки. ${ }^{1}$ На этих рисунках приведены данные, полученные как в стационарных (до $60 \mathrm{kOe}$ ), так и в импульсных (до $250 \mathrm{kOe}$ ) полях. Измерения в импульсных полях для каждого образца проводили при двух „крайних“ (наибольшей и наименьшей) температурах. Сделаем несколько замечаний касательно измерений в импульсных полях. Как известно, температура блокировки SP частиц определяется классическим соотношением НееляБрауна

$$
T_{B}=\mathrm{K} V / \ln \left(\tau / \tau_{0}\right) k_{B},
$$

где $\mathrm{K}$ - константа магнитной анизотропии, $V$ объем частицы, $k_{B}-$ константа Больцмана, $\tau$ и $\tau_{0}$ - характерные времена измерения и релаксации частицы $\left(\tau_{0} \sim 10^{-9}-10^{-11} \mathrm{~s}\right)$. Для квазистатических магнитных измерений обычно используется значение $\tau \sim 10^{1-2} \mathrm{~s}[1]$, что приводит к соотношению $T_{B} \approx \mathrm{K} V / 25 k_{B}$. При измерениях в импульсном поле знаменатель выражения (3) изменяется, что проявлялось, например, в росте коэрцитивной силы наночастиц $\varepsilon-\mathrm{Fe}_{2} \mathrm{O}_{3}$ при уменьшении периода импульса [38]. При $\tau \sim 8 \cdot 10^{-3} \mathrm{~s}$ (время возрастания поля до $\left.250 \mathrm{kOe}\right) T_{B}$ увеличится в $\sim 1.3$ раза по сравнению с квазистатическими магнитными измерениями. В результате $T_{B} \sim 48$ и $\sim 84 \mathrm{~K}$ (рис. 1 ) не превысят температуру измерения в импульсных полях (80 и $110 \mathrm{~K}$ для образцов $3 \mathrm{~h}$ и $240 \mathrm{~h}$ соответственно). При измерениях для этих температур по методике PFM до $250 \mathrm{kOe} \mathrm{наблюдается} \mathrm{небольшой}$ гистерезис в области полей менее $50 \mathrm{kOe}$, что связано с релаксационными процессами частиц с наибольшими температурами блокировки. В этой области полей (до 50-60 kОе) для обработки экспериментальных зависимостей $\mathrm{M}(\mathrm{H})$ использовались данные, полученные в стационарных магнитных полях (VSM).

\footnotetext{
1 Здесь и далее в качестве температуры блокировки $T_{B}$ будем считать температуру максимума зависимости $M(T)_{\mathrm{ZFC}}$ (рис. 1), которая пропорциональна средней температуре блокировки ансабля SP частиц [37] ввиду наличия распределения по размерам, и, следовательно, по значениям $T_{B}$.
} 

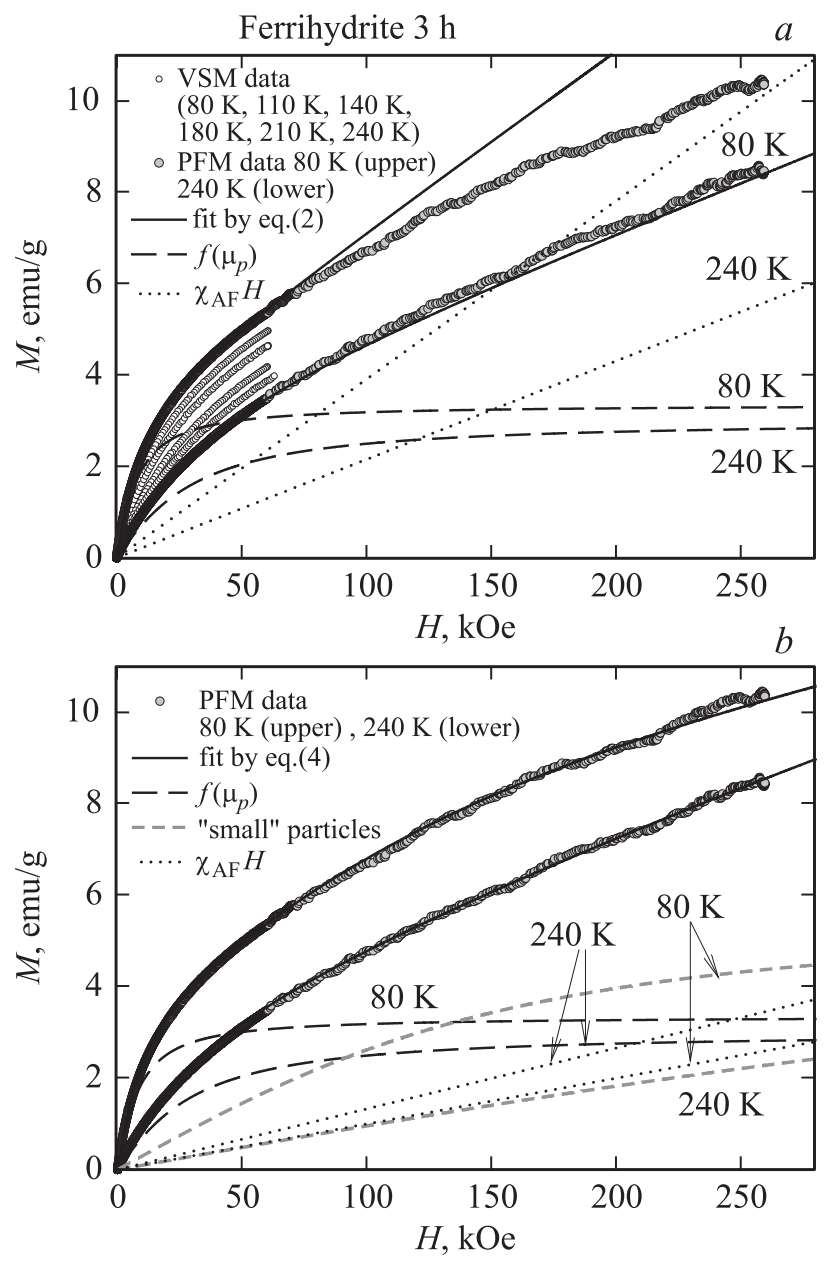

Рис. 2. Кривые намагничивания ферригидрита (образец 3h) в различных температурах и диапазонах магнитного поля. Сплошные линии - результаты наилучшей подгонки данных для диапазона до $60 \mathrm{kOe}$ по выражению (2) (a) и в диапазоне до $250 \mathrm{kOe}$ по выражению (4) (b). Штриховые и пунктирные линии показывают вклады, соответствующие SP частицам с учетом функции распределения $f\left(\mu_{P}\right)$, „малым“ частицам (см. таблицу), а также вклады $\mathrm{AF}$ восприимчивости $\chi_{\mathrm{AF}}(H)$ при указанных температурах.

Сплошные кривые на рис. $2, a$ и $3 a$ иллюстрируют результаты наилучшей подгонки зависимостей $\mathrm{M}(\mathrm{H})$ в диапазоне стационарных полей до $60 \mathrm{kOe}$ по выражению (2), полученные в работе [33]. Фактически подгоночными параметрами при определенной температуре являлись $\chi_{\mathrm{AF}}$ и среднее значение магнитного момента частиц $\left\langle\mu_{P}\right\rangle$ функции распределения $f\left(\mu_{P}\right)$, в качестве которой было использовано логнормальное распределение

$$
f\left(\mu_{P}\right)=\left(\mu_{P} s(2 \pi)^{1 / 2}\right)^{-1} \exp \left\{-\left[\ln \left(\mu_{P} / n\right)\right]^{2} / 2 s^{2}\right\},
$$

где $\left\langle\mu_{P}\right\rangle=n \exp \left(s^{2}\right), s^{2}-$ дисперсия величины $\ln \left(\mu_{P}\right)$, которая так же, как и количество частиц $N_{P}$ в выражении (2), оставалась постоянной при различных температурах. Описываемая процедура позволяет с хорошей точностью описать экспериментальные данные в диапазоне до $60 \mathrm{kOe}$ при различных температурах. На рис. 2, $a$ и $3 a$ также показаны вклады (при указанных температурах), соответствующие двум слагаемым выражения (2) и обозначенные как $f\left(\mu_{P}\right)$ и $\chi_{\mathrm{AF}} H$. Больший наклон зависимости $\chi_{\mathrm{AF}} H$ (второе слагаемое) для меньшей температуры отражает обсуждаемое во Введении „аномальное“ поведение зависимости $\chi_{\mathrm{AF}}(T)$.

В то же время, из рис. $2, a$ и $3, a$ видно, что в области больших полей имеет место серьезное расхождение между экспериментом и подгоночными кривыми. Модельные кривые показывают практически линейный ход в области высоких полей, обусловленный „насыщением“ функции Ланжевена и доминирующим влиянием второго слагаемого выражения (2), в то время как экспериментальные зависимости $\mathrm{M}(\mathrm{H})$ имеют отрицательную кривизну и не выходят на линейный участок в области полей до $250 \mathrm{kOe}$. Подобное наблюдалось в
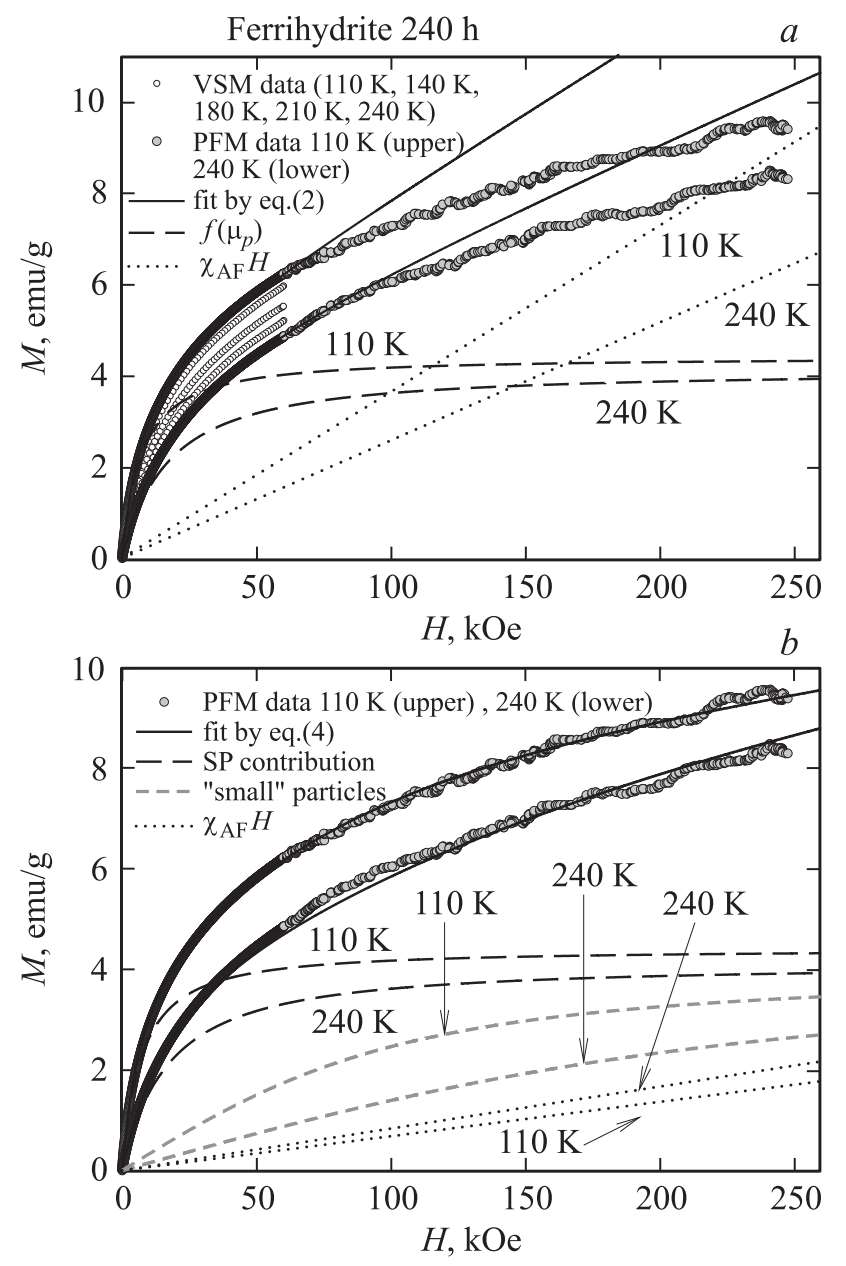

Рис. 3. Кривые намагничивания ферригидрита (образец 240h) при различных температурах и диапазонах магнитного поля. Сплошные линии - результаты наилучшей подгонки данных для диапазона до $60 \mathrm{kOe}$ по выражению $(2)(a)$ и в диапазоне до $250 \mathrm{kOe}$ по выражению (4) (b). Штриховые и пунктирные линии показывают вклады, соответствующие SP частицам с учетом функции распределения $f\left(\mu_{P}\right)$, „малым“ частицам (см. таблицу), а также вклады $\mathrm{AF}$ восприимчивости $\chi_{\mathrm{AF}}(H)$ при указанных температурах. 
работе [4] для ферритина: наклон экспериментальной зависимости $\mathrm{M}(\mathrm{H})$ (производной $d M / d H$ ) становится близким к рассчитанному AF-восприимчивости ферритина только в полях $\sim 500 \mathrm{kOe}$. Для наночастиц $\mathrm{NiO}$ размером $\sim 25 \mathrm{~nm}$ наблюдался линейный рост зависимости $\mathrm{M}(\mathrm{H})$ в диапазоне полей 50-250 kOe [39], т.е. $d M / d H \neq f(H)$ при $H>50 \mathrm{kOe}$.

Итак, путем варьирования величин $\left\langle\mu_{P}\right\rangle, \chi_{\mathrm{AF}}$ и $s^{2}$ функции распределения $f\left(\mu_{P}\right)$ в рамках вышеуказанного подхода [по выражению (2)] не удается достичь согласия экспериментальных и модельных зависимостей $\mathrm{M}(\mathrm{H})$ для ферригидрита во всей области полей. И мы придерживаемся мнения, что наблюдаемый характер экспериментальных зависимостей $\mathrm{M}(\mathrm{H})$ образцов ферригидрита, а именно отсутствие выхода на линейный участок в области полей до $250 \mathrm{kOe}$ обусловлено влиянием малых по величине магнитных моментов (малых частиц), которые не учтены в функции распределения $f\left(\mu_{P}\right)$. Иными словами, зависимость $\mathrm{M}(\mathrm{H})$, обусловленная вкладом этих малых частиц, в диапазоне до $\sim 50-60 \mathrm{kOе}$ является линейной функцией внешнего поля и проявляет характерный ,ланжевеновский“ изгиб с тенденцией выхода на насыщение только в бо́льших полях. Тогда кажущееся уменьшение величины $\chi_{\mathrm{AF}} \mathrm{c}$ ростом температуры, получаемое в результате подгонки по выражениям (1) или (2), является следствием парапроцесса, характерного для SP вклада этих малых магнитных моментов.

Запишем обсуждаемый вклад в виде $M_{\mathrm{SM}} L\left(\mu_{\mathrm{SM}}, H\right)^{2}$, где $\mu_{\mathrm{SM}}-$ магнитный момент, $M_{\mathrm{SM}}-$ намагниченность насыщения, связанная с количеством частиц $N_{\mathrm{SM}}$ в $1 \mathrm{~g}$ образца следующим образом: $M_{\mathrm{SM}}=N_{\mathrm{SM}} \cdot \mu_{\mathrm{SM}}$. Тогда кривая намагничивания будет описываться выражением:

$$
\begin{aligned}
\mathrm{M}(\mathrm{H})= & N_{P} \int_{\mu_{\min }}^{\mu_{\max }} L\left(\mu_{P}, H\right) f\left(\mu_{P}\right) \mu_{P} d \mu_{P} \\
& +M_{\mathrm{SM}} L\left(\mu_{\mathrm{SM}}, H\right)+\chi_{\mathrm{AF}} H .
\end{aligned}
$$

Рис. $2, b$ и $3, b$ иллюстрируют результаты наилучшей подгонки экспериментальных данных по выражению (4) и вклады от „больших“ $\left[f\left(\mu_{P}\right)\right]$, „малых“ частиц и $\mathrm{AF}$ составляющей $\chi_{\mathrm{AF}} H$, соответствующие трем слагаемым правой части выражения (4). В процедуре подгонки экспериментальных данных первое слагаемое выражения (4) оставалось неизменным по отношению к полученным ранее результатам [33], т. е. вклад от „больших“ частиц с функцией распределения $f\left(\mu_{P}\right)$ для данных рис. 2, $a, b$, а также $3, a, b$ одинаков. Варьировались только параметры $M_{\mathrm{SM}}, \mu_{\mathrm{SM}}$ (одинаковые при различных

\footnotetext{
${ }^{2}$ В зависимости $\mathrm{M}(\mathrm{H})$ для магнитных моментов малой величины необходимо принимать во внимание квантование его проекции, что учитывается функцией Бриллюэна. Однако для полученных величин $\mu_{\mathrm{SM}}$ различие между функциями Бриллюэна и Ланжевена незначительное, и значения $\mu_{\mathrm{SM}}$, получаемые при использовании этих функций, отличаются не более чем на $10-15 \%$, что не сказывается на полученных результатах и выводах работы.
}

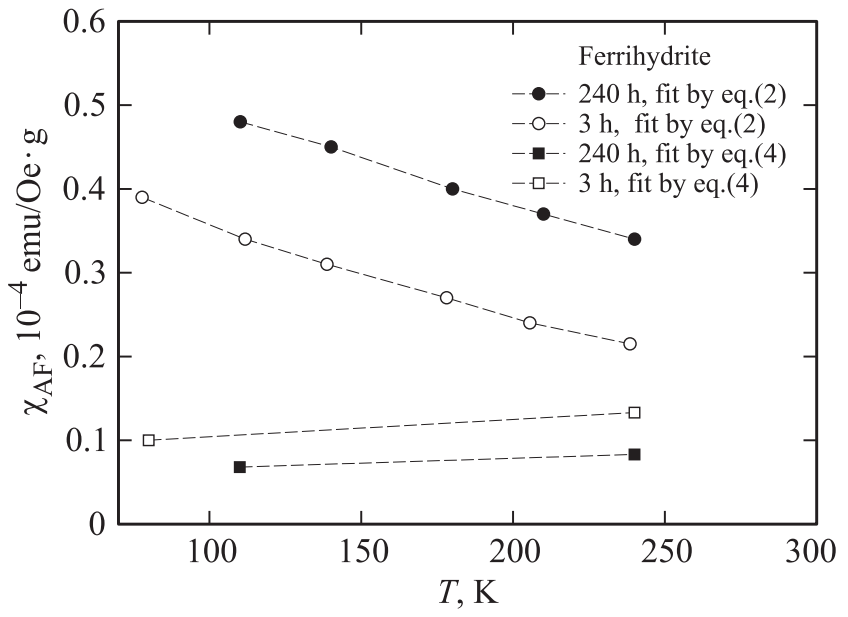

Рис. 4. Величины AF восприимчивости образцов ферригидрита, полученные при обработке изотерм намагничивания в полях до $60 \mathrm{kOe}$ по выражению (2) (рис. 2, $a, 3, a$ ) и до $250 \mathrm{kOe}$ по выражению (4) (рис. 2, $b, 3 b)$.

температурах) и $\chi_{\mathrm{AF}} . \mathrm{B}$ таблице приведены параметры, полученные в результате описанной процедуры подгонки по выражению (4). Как видно из рис. 2, $b$ и $3, b$, учет малых магнитных моментов позволяет достичь хорошего согласия между подгоночными кривыми и экспериментальными зависимостями $\mathrm{M}(\mathrm{H})$ в диапазоне до $250 \mathrm{kOe}$.

Рис. 4 иллюстрирует зависимости $\chi_{\mathrm{AF}}(T)$, полученные ранее из обработки зависимостей $\mathrm{M}(\mathrm{H})$ в диапазоне до $60 \mathrm{kOe}$ по выражению (2) при различных температурах [33], и данные по AF восприимчивости, полученные с учетом вклада малых магнитных моментов по выражению (4) при подгонке эксперимента до $250 \mathrm{kOe}$. С учетом вклада малых частиц получаемые значения $\chi_{\mathrm{AF}}$ меньше по величине, чем при использовании выражения (2), и кроме того, при низких температурах $\chi_{\mathrm{AF}}$ меньше, чем при высоких температурах, что согласуется с классическим поведением AF восприимчивости антиферромагнетика со случайной ориентацией кристаллографических осей в области температур ниже температуры Нееля.

Фактически в используемом подходе была использована двухмодовая функция распределения частиц [40] по магнитным моментам, что указывает на два характерных размера частиц. Размер „больших“ частиц можно получить как непосредственно из микроскопических данных, так и косвенно, используя модельные представления о величине нескомпенсированного магнитного момента AF частицы $\mu_{P}$. Согласно Неелю [41], величина $\mu_{P}$ определяется соотношением:

$$
\mu_{P} \sim J N^{b}
$$

в котором $N-$ количество магнитно-активных атомов в частице, $J$ - магнитный момент атома, а показатель степени $b$ зависит от типа дефектов $(1 / 3 \leq b \leq 2 / 3)$. 
Для ферригидрита и ферритина $[1,15,18-21,23,24,26,42]$ можно считать $b \approx 1 / 2$, что соответствует случайным нарушениям магнитного порядка как на поверхности, так и в объеме частиц. Значения $\langle d\rangle$ (см. таблицу), полученные по выражению

$$
\langle d\rangle \approx d_{\mathrm{Fe}-\mathrm{Fe}} N^{1 / 3} \approx d_{\mathrm{Fe}-\mathrm{Fe}}\left(\left\langle\mu_{P}\right\rangle / 5 \mu_{B}\right)^{2 / 3},
$$

где $d_{\mathrm{Fe}-\mathrm{Fe}}-$ среднее расстояние между атомами железа в ферригидрите $\sim 0.31 \mathrm{~nm}[21]$, хорошо согласуются с данными просвечивающей электронной микроскопии [33].

Что касается „малых“ частиц ${ }^{3}$, оценки которых достаточно проблематично извлечь непосредственно из микроскопических данных, то из выражения (5) при $b \approx 1 / 2$ количество атомов железа в этих частицах будет всего $\sim 10$ (образец 3h) и 60 (образец 240h). Трудно представить частицу с таким малым количеством магнито активных атомов (особенно для первого случая), в которой остается магнитный порядок. Возможно, для экстремально малых частиц величина $b$ [выражение (5)] принимает меньшее значение, подобная гипотеза была предложена в работе [43]. Но в любом случае магнитные моменты порядка $15-40 \mu_{B}$ обусловлены, скорее всего, либо частицами ферригидрита $\sim 1 \mathrm{~nm}$, либо неупорядоченными комплексами атомов железа, кислорода и углерода $[31,44,45]$. При длительном отжиге (образец 240h) эти комплексы могут сформировать частицы ферригидрита, и из таблицы видно, что после отжига уменьшается количество „малых“ частиц, и поскольку возрастает их магнитный момент, то увеличиваются и размеры. Подобный сценарий увеличения размеров частиц в результате отжига, а именно агломерация близко расположенных частиц вытекает из анализа результатов исследования отожженного ферригидрита [24,32-34].

Приведенные оценки размеров „малых“ частиц носят сугубо качественный характер, но присутствие значимого по величине магнитного вклада, обусловленного этими малыми частицами, проявляется в нелинейной зависимости $\mathrm{M}(\mathrm{H})$ в больших полях. На основании проведенного выше анализа можно говорить о двухмодовом типе распределения частиц по размерам, по крайней мере, в исследованном ферригидрите бактериального происхождения. Согласие полученных в настоящей работе экспериментальных данных по намагничиванию в больших полях с результатами работы [4], в которой исследовали ферритин, возможно, указывает на общие черты характера распределения магнитных моментов частиц по размерам в биоферригидрите и ферритине. Кроме того, учет SP поведения малых частиц позволяет заключить, что зависимость $\chi_{\mathrm{AF}}(T)$ для ферригидрита возрастающая с ростом температуры функция, что типично для антиферромагнетика со случайной ориентацией кристаллографических осей, в согласии с выводами работы [4].

\footnotetext{
3 Для „малых“ частиц есть функция распределения по магнитным моментам и размерам, однако обработка экспериментальных результатов с учетом функции распределения приводит к аналогичным результатам, т. е., $\mu_{\mathrm{SM}} \approx\left\langle\mu_{\mathrm{SM}}\right\rangle$, но при этом увеличивается количество подгоночных параметров.
}

\section{4. Заключение}

В данной работе исследованы процессы намагничивания наночастиц антиферромагнитно упорядоченного ферригидрита различных размеров в сильных (до $250 \mathrm{kOe}$ ) магнитных полях. Согласно общепринятому подходу для $\mathrm{AF}$ наночастиц, кривая намагничивания представляет собой суперпозицию вкладов от нескомпенсированных магнитных моментов частиц и антиферромагнитно упорядоченного „ядра“. Однако определение величины $\mathrm{AF}$ вклада, т. е. числовых значений $\mathrm{AF}$ восприимчивости $\chi_{\mathrm{AF}}$, а также ее температурной зависимости очень критично к диапазону магнитных полей, используемых при анализе экспериментальных данных. Если для обычно используемого диапазона полей до $60 \mathrm{kOe}$ (обусловленного стандартизацией установок) зависимость $\chi_{\mathrm{AF}}(T)$ - убывающая с ростом температуры функция, то анализ зависимостей $\mathrm{M}(\mathrm{H})$ в области полей до $250 \mathrm{kOe}$ показал, что зависимость $\chi_{\mathrm{AF}}(T)$ возрастает с ростом температуры. Последнее согласуется с поведением системы $\mathrm{AF}$ частиц со случайной ориентацией кристаллографических осей в области температур, меньших температуры Нееля. Обнаруженный кроссовер вносит серьезные корректировки в физическую интерпретацию экспериментальных кривых намагничивания наночастиц ферригидрита, либо ферритина: наблюдаемое многими авторами уменьшение величины $\chi_{\mathrm{AF}}$ с ростом температуры не связано с эффектом суперантиферромагнетизма, а скорее всего, наведено влиянием малых магнитных моментов, которое можно учесть просто существенно расширив диапазон используемых магнитных полей.

\section{Список литературы}

[1] S. Mørup, D.E. Madsen, C. Fradsen, C.R.H. Bahl, M.F. Hansen. J. Phys.: Condens. Matter 19, 213202 (2007).

[2] D.E. Madsen, S. Mørup, M.F. Hansen. J. Magn. Magn. Mater. 305, 95 (2006).

[3] Yu.L. Raikher, V.I. Stepanov. J. Phys.: Condens. Matter. 20, 204120 (2008).

[4] N.J.O. Silva, A. Millan, F. Palacio, E. Kampert, U. Zeitler, V.S. Amaral. Phys. Rev. B 79, 104405 (2009).

[5] Ю.Л. Райхер, В.И. Степанов. ЖЭТФ 134, 514 (2008).

[6] Ю.Л. Райхер, В.И. Степанов, С.В. Столяр, В.П. Ладыгина, Д.А. Балаев, Л.А. Ищенко, М. Балашою. ФТТ 52, 277 (2010).

[7] A. Punnoose, H. Magnone, M.S. Seehra, J. Bonevich. Phys. Rev. B 64, 174420 (2001).

[8] S.D. Tiwari, K.P. Rajeev. Solid State Commun. 152, 1080 (2012).

S.A. Makhlouf, H. Al-Attar, R.H. Kodama. Solid State Commun. 145, 1 (2008).

[10] A. Punnoose, M.S. Seehra. J. Appl. Phys. 91, 10, 7766 (2002).

[11] A.A. Lepeshev, I.V. Karpov, A.V. Ushakov, D.A. Balaev, A.A. Krasikov, A.A. Dubrovskiy, D.A. Velikanov, M.I. Petrov. J. Supercond. Nov. Magn. 30, 931 (2017)

[12] R.H. Kodama, A.E. Berkowitz. Phys. Rev. B 59, 6321 (1999). 
[13] S. Giri, M. Patra, S. Majumdar. J. Phys.: Condens. Matter 23, 073201 (2011).

[14] A.A. Dubrovskiy, D.A. Balaev, K.A. Shaykhutdinov, O.A. Bayukov, O.N. Pletnev, S.S. Yakushkin, G.M. Bukhtiyarova, O.N. Martyanov. J. Appl. Phys. 118, 213901 (2015).

[15] C. Gilles, P. Bonville, H. Rakoto, J.M. Broto, K.K.W. Wong, S. Mann. J. Magn. Magn. Mater. 241, 430 (2002).

[16] D.A. Balaev, A.A. Dubrovskiy, K.A. Shaykhutdinov, O.A. Bayukov, S.S. Yakushkin, G.A. Bukhtiyarova, O.N. Martyanov. J. Appl. Phys. 114, 163911 (2013).

[17] M.J. Martínez-Pérez, R. De Miguel, C. Carbonera, M. Martínez-Júlvez, A. Lostao, C. Piquer, C. Gómez-Moreno, J. Bartolomé, F. Luis. Nanotechnology 21, 465707 (2010).

[18] N.J.O. Silva, V.S. Amaral, L.D. Carlos. Phys. Rev. B 71, 184408 (2005).

[19] Д.А. Балаев, А.А. Дубровский, А.А. Красиков, С.В. Столяр, Р.С. Исхаков, В.П. Ладыгина, Е.Д. Хилажева. Письма ЖЭТФ 98, 3, 160 (2013).

[20] S.A. Makhlouf, F.T. Parker, A.E. Berkowitz. Phys. Rev. B 55, R14717 (1997).

[21] A. Punnoose, T. Phanthavady, M.S. Seehra, N. Shah, G.P. Huffman. Phys. Rev. B 69, 054425 (2004).

[22] M.S. Seehra, V. Singh, X. Song, S. Bali, E.M. Eyring. J. Phys. Chem. Solids 71, 1362 (2010).

[23] C. Gilles, P. Bonville, K.K.W. Wong, S. Mann. Eur. Phys. J. B 17, 417 (2000).

[24] Д.А. Балаев, А.А. Красиков, А.А. Дубровский, С.В. Семёнов, О.А. Баюков, С.В. Столяр, Р.С. Исхаков, В.П. Ладыгина, Л.А. Ищенко. ЖЭТФ 146, 546 (2014).

[25] Chandni Rani, S.D. Tiwari. J. Magn. Magn. Mater. 385, 272 (2015).

[26] M.S. Seehra, V.S. Babu, A. Manivannan, J.W. Lynn. Phys. Rev. B 61, 3513 (2000).

[27] С.В. Столяр, Р.Н. Ярославцев, Р.С. Исхаков, О.А. Баюков, Д.А. Балаев, А.А. Дубровский, А.А. Красиков, В.П. Ладыгина, А.М. Воротынов, М.Н. Волочаев. ФТТ 59, 3, 538 (2017).

[28] L. Néel. C.R. Acad. Sci. Paris 253, 1286 (1961).

[29] L. Néel, C.R. Acad. Sci. Paris 253, 203(1961).

[30] Ch. Rani, S.D. Tiwari. Physica B 513, 58 (2017).

[31] С.В. Столяр, О.А. Баюков, Ю.Л. Гуревич, В.П. Ладыгина, Р.С. Исхаков, П.П. Пустошилов. Неорг. материалы 43, 6, 725 (2007).

[32] Д.А. Балаев, А.А. Красиков, А.А. Дубровский, О.А. Баюков, С.В. Столяр, Р.С. Исхаков, В.П. Ладыгина, Р.Н. Ярославцев. Письма ЖТФ 41, 14, 88 (2015).

[33] D.A. Balaev, A.A. Krasikov, A.A. Dubrovskiy, S.I. Popkov, S.V. Stolyar, O.A. Bayukov, R.S. Iskhakov, V.P. Ladygina, R.N. Yaroslavtsev. J. Magn. Magn. Mater. 410, 71 (2016).

[34] Д.А. Балаев, А.А. Красиков, А.А. Дубровский, С.В. Семёнов, С.И. Попков, С.В. Столяр, Р.С. Исхаков, В.П. Ладыгина, Р.Н. Ярославцев. ФТТ 58, 2, 280 (2016).

[35] D.A. Balaev, A.A. Krasikov, A.A. Dubrovskiy, S.I. Popkov, S.V. Stolyar, R.S. Iskhakov, V.P. Ladygina, R.N. Yaroslavtsev. J. Appl. Phys. 120, 183903 (2016).

[36] Д.А. Балаев, А.А. Красиков, С.В. Столяр, Р.С. Исхаков, В.П. Ладыгина, Р.Н. Ярославцев, О.А. Баюков, А.М. Воротынов, М.Н. Волочаев, А.А. Дубровский. ФТТ 58, 9, 1724 (2016).

[37] J.C. Denardin, A.L. Brandl, M. Knobel, P. Panissod, A.B. Pakhomov, H. Liu, X.X. Zhang. Phys. Rev. B 65, 064422 (2002).
[38] D.A. Balaev, I.S. Poperechny, A.A. Krasikov, K.A. Shaikhutdinov, A.A. Dubrovskiy, S.I. Popkov, A.D. Balaev, S.S. Yakushkin, G.A. Bukhtiyarova, O.N. Martyanov, Yu.L. Raikher. J. Appl. Phys. 117, 063908 (2015).

[39] Д.А. Балаев, А.А. Дубровский, А.А. Красиков, С.И. Попков, А.Д. Балаев, К.А. Шайхутдинов, В.Л. Кириллов, О.Н. Мартьянов. ФТТ 59, 8, 1524 (2017).

[40] Б.П. Хрусталев, А.Д. Балаев, В.М. Соснин. ФТТ 37, 6, 1676 (1995).

[41] L. Néel. C.R. Acad. Sci. Paris 252, 4075 (1961).

[42] J.G.E. Harris, J.E. Grimaldi, D.D. Awschalom, A. Chiolero, D. Loss. Phys. Rev. B 60, 3453 (1999).

[43] T.H. Lee, K.-Y. Choi, G.-H. Kim, B.J. Suh, Z.H. Jang. Phys. Rev. B 90, 184411 (2014).

[44] M. Balasoiu, S.V. Stolyar, R.S. Iskhakov, L.A. Ischenko, Y.L. Raikher, A.I. Kuklin, O.L. Orelovich, Yu.S. Kovalev, T.S. Kurkin. Rom. Journ. Phys. 55, 7-8, 782 (2010).

[45] С.В. Столяр, О.А. Баюков, В.П. Ладыгина, Р.С. Исхаков, Л.А. Ищенко, В.Ю. Яковчук, К.Г. Добрецов, А.И. Поздняков, О.Е. Пиксина. ФТТ 53, 1, 97 (2011). 